\title{
Eine Berichtigung
}

von August Upmark in Lund.

(Eingegangen am 11. September 1935.)

In meinem Aufsatz über „Elektrische Spitzenwirkungen bei sphärischen Leitern atomarer und subatomarer Größe", ZS. f. Phys. 91, 737-741, 1934, soll Gleichung (8), S. 739, lauten:

$K=-\frac{2(E-e)^{2}}{a^{2}}\left(1-\frac{1}{m-1}\right)=-\frac{2(E-e)^{2}}{a^{2}}\left(1-\frac{2}{n-1}+\frac{1}{2 n-1}\right)$. $P_{A}=\frac{2 m(E-e)}{a}\left(1-\frac{1}{m-1}\right)$ wird nämlich im Abstand $a$, wo die Zentren von $B$ und $C$ liegen, $m$-mal kleiner und

$$
P_{B}=P_{C}=-\frac{n(E-e)}{a}\left(1-\frac{2}{n-1}+\frac{1}{2 n-1}\right)
$$

in dem Abstand des Zentrums von $A$ numerisch n-mal kleiner.

Der Koeffizient $1-\frac{1}{m-1}=1-\frac{2}{n-1}+\frac{1}{2 n-1}$ ist also nur für $m=n=\infty$ gleich 1 . 